\title{
An Immunoassay Platform Based on CMOS Hall Sensors
}

\author{
Turgut Aytur, P. Robert Beatty ${ }^{1}$, Bernhard Boser \\ Dept. of Electrical Engineering \& Computer Sciences, ${ }^{1}$ Dept. of Molecular \& Cellular Biology \\ University of California, Berkeley, CA 94720 \\ Mekhail Anwar \\ Dept. of Electrical Engineering and Computer Sciences \\ Massachusetts Institute of Technology \\ Cambridge, MA 02139 \\ Tomohiro Ishikawa \\ System Technology Research Center \\ Sharp Corporation \\ Chiba, Japan
}

\section{ABSTRACT}

We describe an immunoassay utilizing standard CMOS technology. An array of Hall sensors is used to detect the magnetic beads that serve as the assay signal. Electrical and magnetic modulation is employed to improve the sensitivity of the sensors. The devices receive two post-processing steps to improve sensitivity and biocompatibility. We have fabricated prototype devices using a $0.25-\mu \mathrm{m}$ BiCMOS process, and have successfully detected anti-Hu IgG antibody at a concentration of $200 \mathrm{pM}$.

\section{INTRODUCTION}

Diagnosis is an essential tool in the health care industry. The role of diagnosis is expanding, particularly within the context of screening and prevention. Infectious diseases are a major cause of death in the world, with HIV/AIDS, tuberculosis, and malaria responsible for approximately 5.7 million deaths in 1998 [1]. Rapid diagnosis is essential during epidemics for fast treatment and containment.

A dominant technology in diagnostics is the Enzyme-Linked Immunosorbent Assay (ELISA). Immunoassays, such as ELISA, are diagnostic tools that rely on the highly specific interaction of antibody binding to cognate antigen. In the ELISA, the detection of unknown antibody or antigen is signaled through an enzymatic label that activates a dye. ELISAs can be administered in a doctor's office or hospital environment, allowing for cost effective diagnosis of a broad range of diseases. However, most ELISA tests require a basic laboratory environment and some staff training. For many infectious diseases, a rapid, precise quantitative measurement is required for accurate diagnosis, which is rarely possible in the field. Therefore, improvements in diagnosis should focus on rapid, cost-effective in-field or point-ofcare testing.

\section{Magnetically-Labeled Assays}

The limitations in ELISA for quantified point-of-care diagnostics stem mostly from the enzymatic label. Recently, the use of micron-scale magnetic beads as labels has been described $[2,3,4]$. There are many advantages gained from using a magnetic label. First, there are no comparable sources of magnetic signal in biology, so the background is intrinsically low. Second, magnetic beads can be used to manipulate biological systems The beads are specifically superparamagnetic, as they have very low remnance. When placed in a magnetic field, however, they generate an induced magnetic field. The magnetic beads can be made biologically active by coating the polystyrene encapsulation.

Travel support was generously provided by the Transducers Research Foundation, and the DARPA MEMS and DARPA BioFlips Programs.

The magnetic label requires a different detection system than ELISA. The first reports of assays utilizing magnetic labels employed superconducting quantum interference devices as sensors [2]. These devices are highly sensitive to magnetic fields, but are not portable. More recently, a device has been developed based on giant magneto resistor (GMR) technology $[3,4]$. GMR devices are highly sensitive, and are now in mass production in computer disk-drive read heads. A simplified protocol for an assay involving this device is shown in Figure 1.
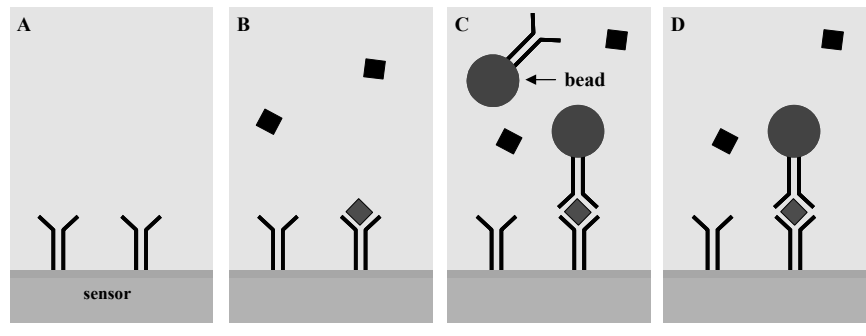

Figure 1. Simplified magnetically-labeled assay.

The gold surface received a coating protein. The test liquid was added (Figure 1b). Next, magnetic beads coated with an appropriate antibody against the target antigen were added (Figure 1c). Magnetic beads far from the sensor but still in solution will not affect the result. This assay used magnetic washing, where unbound beads were pulled from the sensor by a magnetic field (Figure 1d). An external magnetic field normal to the sensor surface was then applied. The induced field generated by bound beads was measured as a resistance change in the GMR sensor. The amount of change in resistance corresponds to the number of beads bound. This device has single magnetic bead sensitivity.

Improvements in cost and manufacturability of the magnetic sensors may broaden the applicability of magnetically-labeled assays. CMOS is the most widely manufactured semiconductor technology. It is an attractive substrate for MEMS devices due to manufacturing ease and potential as an intelligent substrate. The sensor can be easily integrated with other functionality. The small size of the sensor chips described in this paper implies that the microfabrication costs will not dominate the assay expense. It is therefore beneficial to utilize CMOS for the sensing technology.

\section{THEORY}

Hall sensors can be operated in current or voltage mode. A particular example of a current-mode device that exploits the MOS transistor structure is the dual-drain Hall FET. The differential output current of this device can be expressed as [5],

$$
i_{d}=\frac{L}{2 W} \mu_{H} G B_{z} I_{D S}
$$


where $W$ and $L$ are the channel width and length, respectively, of the device, $\mu_{H}$ is the Hall mobility, and $G$ is a geometric constant that accounts for current confinement at the device boundaries. In the low magnetic fields of this application, the Hall mobility is well represented by the carrier mobility. For a long-channel device operating in saturation, Eq. 1 can be rewritten as,

$$
i_{d} \cong \mu_{e f f}^{2} / 4 G B_{z} C_{o x}\left(V_{g s}-V_{t}\right)^{2}
$$

where $\mu_{\text {eff }}$ is the effective carrier mobility.

\section{Device Scaling}

In the analysis above, the applied field is assumed to be uniform and normal to the sensor surface. Magnetic beads produce a local, non-uniform field when placed in an external field. This field is well modelled by a magnetic dipole equation. The peak signal decays with cubic dependence on the height from the sensor plane. A simulation of the induced magnetic field, measured normal to a plane $5 \mu \mathrm{m}$ below the center of a $5-\mu \mathrm{m}$ bead, is shown in Figure 2.

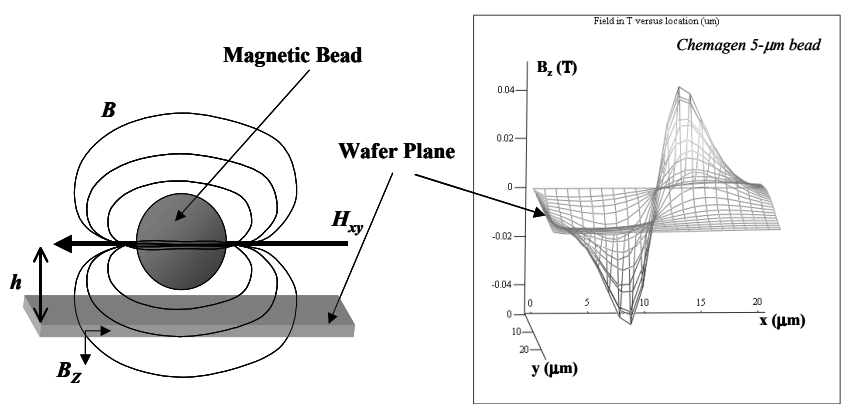

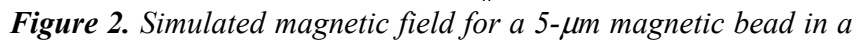
$35-k A / m$ field, 5- $\mu m$ for the surface.

The total field integrates to zero, since the field strength is antisymmetric about the y-axis. Therefore, a hall-sensor device that is much larger than the bead will have a smaller signal than a correctly scaled device. Consider the hypothetical situation shown in Figure 3. In these devices, the magnetic field is zero outside the shaded strip, and finite in it. The current bends in the region with field, as predicted, but tends to redistribute in the no field region. From this illustration, it is clear that the Hall sensor device size should be scaled according the bead size. The minimum size of the sensor is limited by lithographic constraints.
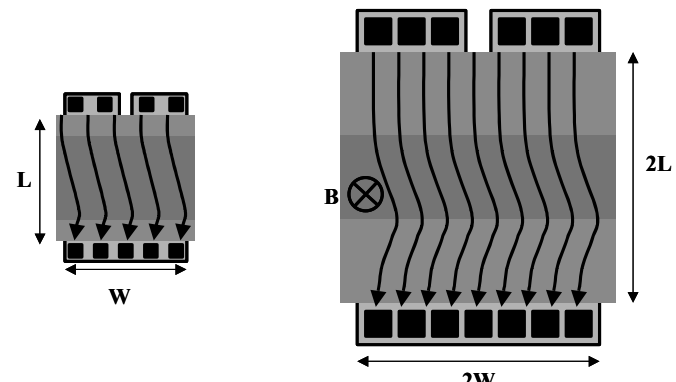

Figure 3. Illustration of current lines for two device sizes in the same non-uniform magnetic field. The larger device has a reduced output signal due to charge redistribution.
Flicker Noise

CMOS devices have poor low-frequency noise properties due to flicker or $1 / \mathrm{f}$ noise. The noise spectral density, as the name suggests, is inversely proportional to frequency. The consequence of this is that signals at DC do not benefit from narrowing of the noise bandwidth, eliminating the trade-off between bandwidth and Signal-to-Noise Ratio (SNR). Thus, there exists a minimum detectable signal, independent of noise bandwidth.

For conventional magnetic sensing applications, the signal frequency is either not known or assumed to be at DC. However, in this application the excitation frequency of the external magnetic field is limited only by practical constraints of electromagnets. This improves the signal detection in two ways: First, a band-pass filter can be employed to restore the trade-off between bandwidth and SNR. Second, the signal can be moved to a frequency of lower spectral noise density. Unfortunately, there is still approximately one order of magnitude difference between the flicker noise corner frequency of the MOS hall device and the maximum frequency to practically operate the electromagnetic excitation. To overcome this, we combine electrical modulation and magnetic modulation, as represented in Figure 4.
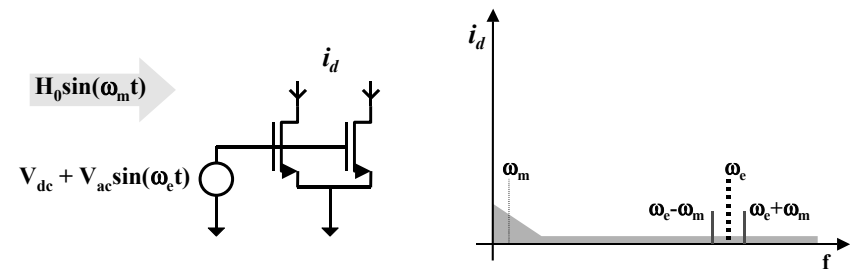

Figure 4. Illustration of magnetic and electrical modulation applied to the dual-drain NMOS sensor device. The modulated output signal appears at frequencies $\omega_{e} \pm \omega_{m}$,

The gate-to-source voltage and magnetic field are applied as:

$$
\begin{aligned}
& V_{g S}(t)=V_{D C}+V_{A C} \sin \left(\omega_{e} t\right) \\
& H_{x y}(t)=H_{0} \sin \left(\omega_{m} t\right)
\end{aligned}
$$

Where $\omega_{e}$ and $\omega_{m}$ are the electrical and magnetic modulation frequencies, respectively. Combining eq. (2) with eqs. (3) and (4) defines the modulated output spectrum. In addition to DC and higher order terms, the differential drain current will contain the term:

$$
i_{d} \propto \sin \left(\omega_{e} \pm \omega_{m}\right)
$$

The electrical modulation frequency can be selected such that thermal noise, rather than flicker noise, dominates. The magnetic modulation is desirable to separate the wanted signal from carrier leakage (dashed line in Figure 4). Carrier leakage results from a variety sources, including device leakage and parasitic coupling, and results in a limit on the minimum detectable signal.

\section{DESIGN AND FABRICATION}

Sensor Chip

The sensor chips were fabricated in Agere Systems (formerly Lucent Technologies-Microelectronics) $0.25-\mu \mathrm{m}$ single-poly 5- 
metal BiCMOS process. Each Hall device is implemented as a 6$\mu \mathrm{m} \times 6-\mu \mathrm{m}$ dual-drain NMOS device. The drains are separated by a $1-\mu \mathrm{m} \times 1-\mu \mathrm{m}$ field-oxide region. Each sensor consists of two matched devices that source current in opposite directions (Figure 5), so that uniform magnetic fields are rejected.

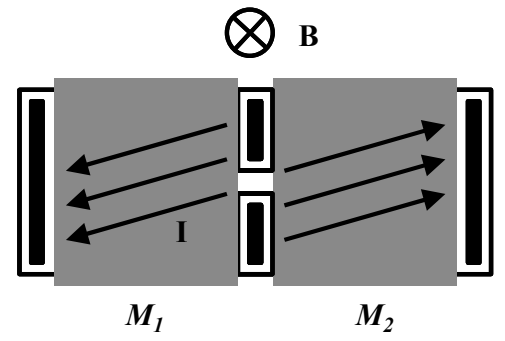

Figure 5. Two Hall devices source current in opposite directions, reducing uniform fields by 30-40 dB.

This reduces the leakage signal from the excitation field by 30 $40 \mathrm{~dB}$. This leakage signal occurs due to misalignment of the excitation field with the sensor surface.

The sensor array consists of $32 \times 8$ sensor elements. Figure 6 shows a portion of the sensor array.

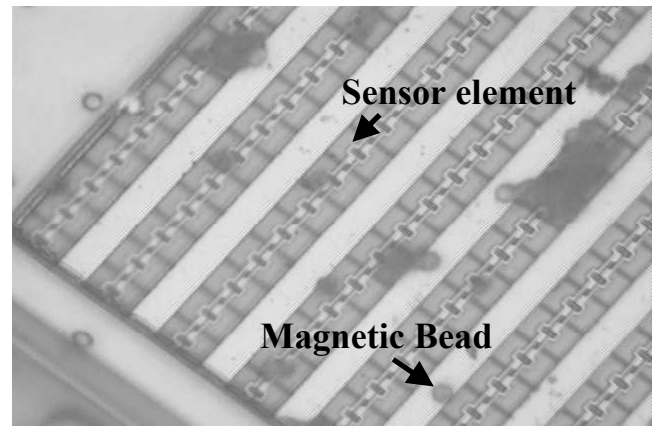

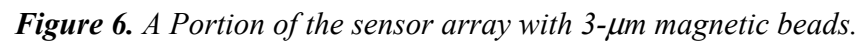
The Cr/Au layer was not applied to allow viewing of sensor detail.

Each element is addressable via a shift register. The drain current of the selected sensing device is converted to a voltage before being amplified by a bipolar tranconductance amplifier. This balanced, current-mode output is sent off chip.

Due to a chip area restriction of $1 \mathrm{~mm} \times 1 \mathrm{~mm}$, additional metalization post-processing was necessary to move bond pads away from the sensing area. This allows wire bonding to the chip outside of the fluid vessel. This part of the post-processing will not be described, as future prototypes will accomplish this with the standard metallization.

Devices are post-processed in subsections of the initial 8 " wafer. First, the silicon dioxide above the sensor area is thinned by plasma etching. This reduces the distance from a magnetic bead to the sensor surface, increasing the signal. The etch depth is controlled by comparison with etch reference marks implemented in the standard metallization, resulting in a final oxide thickness of approximately $2 \mu \mathrm{m}$. Next, a thin layer $(50 \mathrm{~nm} / 150 \mathrm{~nm})$ of $\mathrm{Cr} / \mathrm{Au}$ is patterned over the sensor area using lift-off. Other materials were tested for protein adsorption, including $\mathrm{Ti}$ and $\mathrm{Cu}$, but were found to be less effective than $\mathrm{Au}$. However, $\mathrm{Cu}$ may represent a significant advantage in processing simplicity for CMOS processes that use $\mathrm{Cu}$ for metalization.
Packaging and Experimental Setup

Processed chips, including metallization extension, are assembled as drawn in Figure 7.

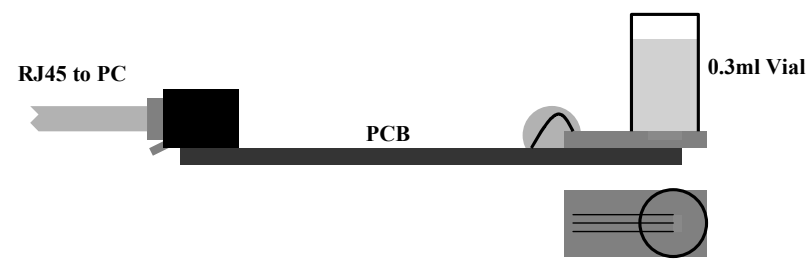

Figure 7. Simplified assembly diagram of sensor chip with fluid container and printed circuit board.

$9 \mathrm{~mm} \times 15 \mathrm{~mm}$ chips are mounted on a $15-\mathrm{cm}$ long PCB. A $300-\mu \mathrm{L}$ polystyrene vial is inverted and epoxied to the silicon chip. A small hole is predrilled in the vial to allow fluid entry. The PCB connects to an analog processing board via RJ45 connector. A 1$\mathrm{kHz}, 30-\mathrm{kA} / \mathrm{m}$ magnetic field is applied parallel to the sensor surface by a custom C-core electromagnet (Figure 8).

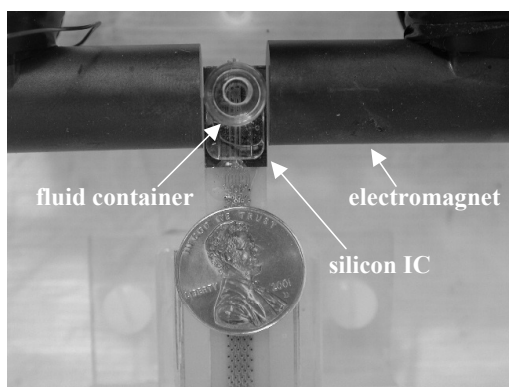

Figure 8. Sensor chip and assembly mounted in the C-core electromagnet.

This magnet is mounted on rubber bushings so that it can be adjusted to reduce leakage normal to the sensor surface. The 250$\mathrm{kHz}$ electrical modulation signal is sinusoidal and is applied to both the gate of the active sensing device and the input of the mixer IC. The balanced, current-mode output of the post amplifier is converted to a voltage on the analog processing PCB and amplified by instrumentation amplifiers. A simplified diagram of the signal processing is shown in Figure 9. Data is processed automatically in Matlab (The Mathworks, Natick, MA). The signal is digitally demodulated into in-phase and quadrature baseband components. These baseband signals are filtered by a FIR low-pass filter before reconstruction into polar form.

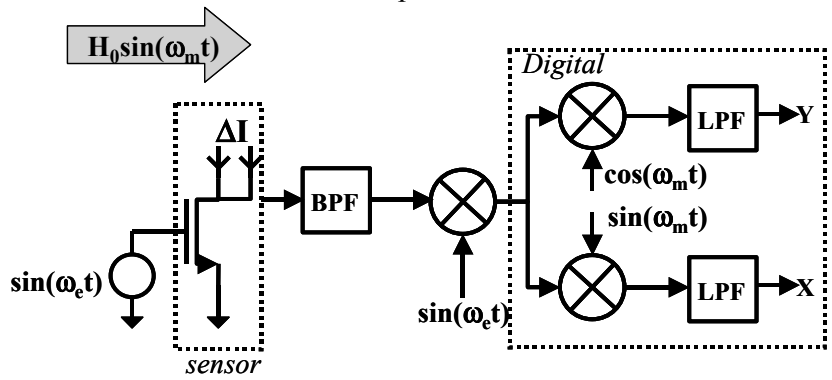

Figure 9. Simplified diagram of the signal processing path. Digital signal processing is performed in software.

The noise bandwidth can be adjusted by controlling the FIR filter bandwidth. 
RESULTS

\section{Anti-Hu IgG Assay}

The sensor chip surfaces were coated overnight with $10 \mu \mathrm{g} / \mathrm{ml}$ human IgG diluted in phosphate buffered saline (PBS). Surfaces were blocked with 3\% Non-Fat Dry Milk for 1 hour and washed 3 times in PBS with $0.5 \%$ Tween-20 (PBS-T). Either biotinylated goat anti-human IgG (200pM) or biotinylated goat anti-mouse IgG (as a control) (Sigma Aldrich, St. Louis, MO) was added to separate vials and incubated for 30 minutes. The samples were then washed 3 times with PBS and streptavidin-coated magnetic beads (5-8um diameter, Chemegen, Germany) diluted 1:200 to $125 \mathrm{ug} / \mathrm{ml}$ were added and allowed to settle for 20 minutes. A rareearth magnet was placed approximately $8 \mathrm{~mm}$ above the sensor surface for 60 seconds before measurement. The magnet position was determined empirically. The sample was then placed in the measurement system. Figure 10 shows the response from the first 96 sensor elements for the goat anti-human IgG shown in grey and the goat anti-mouse IgG shown in black.

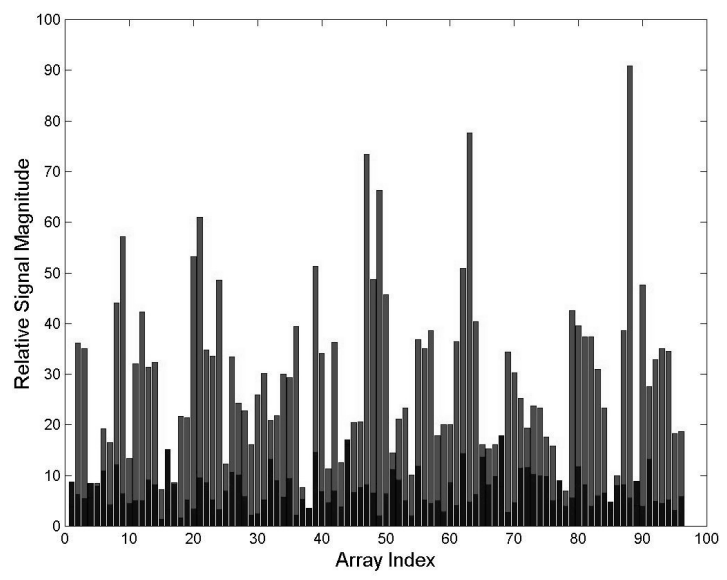

Figure 10. Relative output signal magnitude vs. array element index for anti-Hu IgG target protein (grey) and control (black). The average signal-to-noise ratio is approximately $13 \mathrm{~dB}$.

This data was collected prior to the use of electrical modulation. Recent experiments indicate that use of electrical modulation improves SNR by approximately $10-20 \mathrm{~dB}$. This improvement in SNR can be used to reduce the scan time for the array. Scanning all 256 elements takes approximately 2 minutes. The scanning time for the array becomes important if the device is scanned repeatedly while the magnetic washing force is ramped. This washing method may provide additional information about assay binding characteristics.

\section{CONCLUSIONS}

We have demonstrated an immunoassay platform with clinically relevant sensitivity, fabricated in a CMOS process. The noise and sensitivity limitations that traditionally limit the applicability of CMOS Hall sensors have been mitigated through architectural and signal processing techniques. With these improvements, the CMOS substrate may provide a cost-effective and easily manufactured platform for diagnostics. Furthermore, the proposed assay platform is potentially compact and automated, making it applicable to in-field applications.
The authors would like to thank Agere Systems for microfabrication support and Prof. Eva Harris for discussion and laboratory resources.

\section{REFERENCES}

1. World Health Organization Infectious Disease Report, 1999. 2. R. Kotitz, et al, "SQUID based remnance measurements for immunoassays", IEEE Trans. on Applied Superconductivity, 7, 36 (1997).

3. D. Baselt, et al, "A biosensor based on magnetoresistance technology", Biosensors and Bioelectronics, 13, 791 (1998).

4. R.L. Edelstein, et al, "The BARC biosensor applied to the detection of biological warfare agents", Biosensors and Bioelectronics, 14, 805 (2000).

5. R. S. Popovic, Hall Effect Devices, New York, IOP ltd, 1991. 